\title{
XII Convegno della Società Canadese per gli Studi di Italianistica
}

Nell'agreste tranquillità dell'Università di Guelph si è svolto il XII Convegno della Società Canadese per gli Studi d'Italianistica tra il 3 e il 5 giugno 1984, nell'ambito delle Società Accademiche Canadesi. Data la prossimità dell'università alle città di Toronto e Hamilton, o forse proprio a causa di essa, l'afflusso dei partecipanti è stato piuttosto saltuario e meno numeroso di quel che si pensava sebbene il programma, suddiviso nelle abituali quattro sezioni, si presentasse ricco, vario e attraente.

Molto diversificata la sezione sul Medioevo presieduta da Domenico Pietropaolo (University of Toronto). In "A ciascun'alma presa e gentil core. . . Considerazioni per l'interpretazione del primo sonetto de La Vita Nuova" Gabriele Erasmi (McMaster University), seguendo una vena interpretativa che sta esplorando da qualche tempo, ha proposto una rilettura dei sonetti di risposta a quello dantesco. Da essa risulterebbe che questo sonetto originariamente non sarebbe stato un presentimento per la morte di Beatrice ma una elaborata allegoria mistica. Cino l'avrebbe interpretata convenzionalmente. Il Cavalcanti, invece, avrebbe intuito in essa eruditi elementi della cosmologia avicenniana e averroistica senza peraltro cogliere importanti idee che avvicinano Dante all'amore mistico di Ahmab Ghazali. Dante da Maiano, meno erudito, avrebbe visto nel sonetto solo un'inaudita esasperazione dei sensi al di fuori di ogni tradizione casistica amorosa. In "I miglior fabbri del parlar materno, Arnaut Daniel e Dante: un'analisi tecnica e tematica delle loro sestine" Filippo Salvatore (Concordia University), ha spiegato come la complessità psicologica del fin amors provenzale trovi riscontro nel trobar clus di cui Arnaut Daniel è il piú tipico rappresentante. Ha poi illustrato lo schema della retrogradatio cruciata delle parole-rime della sestina - una forma di canzone senza piedi né volte, inventata dal Daniel - e ne ha sottolineato il rigore logico. Per il Salvatore Dante avrebbe visto nel trovatore "il miglior fabbro del parlar materno" e lo avrebbe emulato nelle due 
sestine delle "rime petrose" - la fase apertamente sperimentale del suo tirocinio. Ha concluso affermando che il dolce stil novo è stato per Dante una conquista personale, piú che di scuola, perché la loda e la terza rima costituiscono novum aliquid atque intentatum in poesia. Albert E. Wingell (University of Toronto), in "The Sloth of Statius: Purgatorio XXII" ha notato che solo recentemente i critici hanno individuato un "modello" per la storia di Stazio narrata da Dante. Si tratterebbe di quella di Marius Victorinus nella versione elaborata da Sant'Agostino nelle Confessioni. Per lui, sebbene i due racconti abbiano un finale diverso, il mero confronto risolve alcuni dei problemi che i critici hanno notato a proposito del personaggio "Stazio" creato da Dante. Inoltre esso darebbe dei dati di supporto per un'interpretazione autobiografica: anche nella sua acedia Stazio è una figura di Dante stesso. In "Coreferenza linguistica nei racconti del Novellino" Jana VizmullerZocco (University of Toronto), ha mostrato che la testualità nei racconti del Novellino riflette due forze antitetiche: una che si esprime nell'eccessiva preoccupazione di individuare i personaggi mediante la coreferenza, l'altra di lasciare l'interpretazione delle relazioni logico-semantiche all'inferenza. Ed ha concluso che questo fenomeno è dovuto al particolare stadio nello sviluppo del volgare, e non alla mancanza di sensibilità artistica dell'autore. Charles K. Langford (Concordia University), in "La serie numerica di Leonardo Pisano" dopo aver stabilito che Leonardo Pisano, detto Fibonacci, nel suo Liber Abbaci (1202) espone la somma di tutta la scienza matematica araba dell'epoca e, ancor piú, presenta spunti geniali che porteranno alla moderna scienza del calcolo, ha continuato col dire che Fibonacci nel suo tempo fu un incompreso e tale restò per oltre due secoli. Il Langford ha concluso poi con l'asserire che sebbene il Pisano sia ricordato come l'autore della prima serie numerica, in lui si ritrova di piú: i suoi scritti rivelano i prodromi dello spirito rinascimentale.

Varia sia per la tematica come per gli autori trattati la sezione sul Rinascimento e Barocco presieduta da Filippo Salvatore (Concordia University). In "The Editio Princeps of Boccaccio's Commentary on the Divine Comedy" Domenico Pietropaolo (University of Toronto), servendosi di materiali a stampa e di diversi documenti inediti, ha tracciato la storia della travagliata prima edizione del commento dantesco di Boccaccio datata Firenze 1724 ma pubblicata a Napoli alla fine del 1725 . Ha notato come essa sia stata curata da Anton Maria Biscioni e non da Anton Maria Salvini o da Anton Francesco Marmi ai quali è invece attribuita dalla tradizione editoriale. Ha poi concluso con l'osservare che buona parte dei 
numerosi "errori" della editio princeps non sono dovuti ad una trascrizione imprecisa del testo, come comunemente si crede, ma ad un tentativo ad opera dello stesso Biscioni di migliorarlo filologicamente, riducendolo ad un suo ideale di "buona ortografia." Ernesto Virgulti (University of Toronto), in "I nuovi volti del Decameron" ha proposto una revisione critica dei piú recenti studi sull'opera del Boccaccio, rilevandone non solo i contributi ma anche le metodologie teoriche che ne sono alla base. Dopo aver analizzato le implicazioni, le lacune e l'influenza su susseguenti analisi strutturali della Grammaire du 'Décaméron' di T. Todorov, il Virgulti ha passato in rassegna gli studi narratologico-linguistici delle novelle di Cesare Segre. Infine ha paragonato e contrastato la lettura in chiave di "meta-romanzo" di Guido Almansi, sia con questi ultimi sia con letture piú tradizionali. Rita Belladonna (University of Western Ontario), in "An Unknown Source of Bernardino Ochino: the Dialoghi Sette and Ubertino da Casale" ha osservato che nel suo Dialogo del Ladrone in Croce l'Ochino si serví largamente di brani tratti dai capitoli XIII e XIV dell'Arbor Vitae Crucifixae Jesu (1305) opera profetica di Ubertino da Casale, figura chiave del movimento spirituale francescano. Ha aggiunto che l'uso di passi riferentisi alla giustificazione per sola fede permise all'Ochino di costruire abilmente un sottile discorso a due livelli diversi. I filoprotestanti italiani potevano cogliervi idee legate all'evangelismo del tempo, mentre le stesse idee non erano probabilmente recepite dai lettori meno sensibili alle questioni religiose dei primi decenni del Cinquecento. In "Eros e follia nella trattatistica del Seicento" Massimo Ciavolella (Carleton University), tramite lo studio di opere esemplari del 1500 e 1600 - in particolare il Commentarium in Convivium Plantonis de Amore, il Liber de vita, di Marsilio Ficino, e il commento di Marco Aurelio Severino all'edizione delle rime di Giovanni della Casa stampato nel 1694 - , analizza il rapporto eros-follia, postulando un cambiamento radicale nel codice concettuale del linguaggio di eros. Secondo il Ciavolella, tra il 1500 e il 1600 la concezione di eros - sotto la spinta del crescente razionalismo - perde la predominante componente metafisica per essere considerato fenomeno prettamente fisiologico, una deviazione dovuta ad un desiderio eccessivo e malsano (cioè antisociale) e pertanto appartenente agli stati patologici dell'individuo. In "La Sicilia nel Settecento e la polemica gentiliana" Salvatore Bancheri (University of Ottawa), asserisce che Il tramonto della cultura siciliana (1917) di Giovanni Gentile, che presenta una Sicilia settecentesca retriva e isolata dal movimento culturale europeo, è il punto di partenza per una valutazione critica dell'atmo- 
sfera culturale siciliana del secolo decimottavo. Questo giudizio del Gentile generò un'accesa polemica tra coloro che vedevano la Sicilia "sequestrata" dal moto culturale europeo e coloro che invece consideravano l'isola come una "piccola Francia illuministica e rivoluzionaria." Il Bancheri ha concluso col dire che d'ora in avanti gli avvenimenti politici, sociali e culturali della Sicilia del Settecento saranno sempre esaminati alla luce di questa polemica.

Ricca e stimolante, anche per la presenza dello scrittore Libero Bigiaretti, la sezione sul periodo moderno e contemporaneo diretta da Vera Golini (University of Waterloo). Guido Pugliese (University of Toronto), ne "Il discorso delle immagini ne I Promessi Sposi," ha notato come in passato la critica ha studiato le immagini manzoniane singolarmente per metterne in rilievo soprattutto la loro perspicuità, naturalezza e convenienza, e come piú di recente sono apparsi articoli in cui l'uso del linguaggio figurato è stato riportato a principi di ordine retorico-simbolico. Ha poi sostenuto che non solo l'uso delle immagini è informato a una "rettorica fine, di buon gusto," ma anche che le immagini hanno un proprio disegno strutturale e un proprio discorso - discorso cui tenderà alla fine l'elemento concettuale e quello della fabula. Infine ha proposto una nuova interpretazione dell'Introduzione il cui valore si ritrova nelle immagini ivi contenute - guerra, tempo, labirinto, morte - che poi risultano essere le figure portanti del romanzo. In "Scienza, progresso e fede nell'opera di Giacomo Zanelia" Giuliana Sanguinetti Katz (University of Toronto), ha sostenuto che in tutta l'opera dello Zanella corre una tensione dovuta al suo atteggiamento ambiguo verso la scienza, vista come fautrice di progresso ma anche come guida pericolosa che può corrompere l'uomo e condurlo alla rovina, se non è moderata dalla sapienza e dalla fede. Ha poi concluso che i sentimenti del poeta, pur riflettendo chiaramente i problemi del tempo, sono legati a contrasti interni dell'autore e ad esperienze emotive che risalgono al periodo dell'infanzia. In particolare le profonde depressioni di cui lo Zanella soffrí tutta la sua vita possono spiegare la sua visione "divisa" della scienza e dell'umanità. Claudia Persi Haines (Carleton University), in "From the Recording of Memory to the Absence of Memory" ha presentato una sua ricerca preliminare basata sull'ipotesi di possibili analogie fra l'analisi della narrativa in prima persona e l'analisi di strategie di composizione in linguistica applicata. Ha sostenuto il concetto di scrittura come scoperta ed ha analizzato tre fasi diverse del processo di scrittura tramite tre personaggi pirandelliani, scrittori "umoristici," colti nell'atto di scrivere. In "Meta-fiction and Meta-lies in The Lie of Moravia: a Com- 
ment on Post-Modernism" Rocco Capozzi (University of Toronto), ha descritto L'Attenzione (1965) di Moravia come un meta-romanzo che illustra la "verità" ontologica della narrativa e delle metamorfosi del protagonista-narratore nell'atto dello scrivere. Per Capozzi la fusione di verità e menzogne da parte dell'autore mostra anche come le "menzogne narrative" (e la novellistica in generale) siano degli eccellenti mezzi che rivelano la "verità" circa il narratore, il romanzo e la realtà in generale. Infine lo scrittore Libero Bigiaretti (Roma), ci ha parlato di quello che per lui è stato l'avvenimento letterario italiano piú notevole dell'anno, cioè la pubblicazione dei testi che Giuseppe Ungaretti scrisse tra il 1937 e il 1942 per preparare le sue lezioni di letteratura italiana all'Università di San Paolo nel Brasile. Da questi testi risulta che il Poeta e il Professore si trovano a convivere senza difficoltà "anzi con i modi di una intesa cordiale e armonica." [Per un discorso piú ampio vedere la recensione del Bigiaretti al volume stampata in questo stesso numero]. Dopo averci invitati alla lettura dei testi stessi il Bigiaretti ci ha parlato dei suoi rapporti personali con lo scrittore alessandrino e li ha descritti come "cordialissimi" di una "amicizia profonda e sincera," ed ha narrato anche degli aneddoti dai quali la figura del poeta è apparsa in una luce piú calda, piú umana ben diversa da quella che emerge dai testi o dalle raccolte di poesie.

La sezione dedicata alla pedagogia e alla linguistica applicata diretta da Diego Bastianutti (Queen's University), è stata anche piuttosto ricca e nutrita. In "A Communicative Syllabus for the Teaching of Italian" Gabriella Colussi (York University), dopo aver notato come l'approccio comunicativo nell'insegnamento di una lingua straniera abbia aperto le porte a tutto un nuovo modo di pensare, ha fatto osservare come esso ancora non sia stato applicato nei libri di testo disponibili. Pertanto in attesa di una produzione di testi di base comunicativa, ha proposto una soluzione provvisoria: applicare un modello di organizzazione del sillabo comunicativo al sillabo strutturale-situazionale che predomina nei testi attuali. Per la Colussi questo sarebbe già un passo avanti nell'avvicinare gli studenti che studiano l'italiano come lingua straniera alla realtà comunicativa della lingua stessa. Claudia Persi Haines (Carleton University), in "Subject Matter as Comprehensive Input for Beginners" ha parlato di un esperimento condotto con gruppi di studenti di architettura della sua università in tre fasi diverse ed ha proposto un modulo propedeutico per l'apprendimento di una seconda lingua (in questo caso l'italiano) basato sulla competenza degli studenti nella loro disciplina (in questo caso l'architettura). In "Per una definizione degli errori semantici" 
Nicoletta Villa (York University), ha voluto cercare di rimediare ad un problema epistemologico riscontrato nell'analisi degli errori. Si tratta della definizione stessa del termine errore, che, di fatto, è alla base di tutta la teoria. Ha sostenuto che finora ci si è basati piú su un'intuizione a proposito del concetto di errore che su una definizione precisa. Pertanto ha proposto due iter per poter definire ed identificare gli errori semantici che non sono stati, fino al momento attuale, trattati a fondo dagli studiosi dell'analisi degli errori. Infine ha avanzato una proposta di definizione specifica e comprensiva di aspetti situazionali come base delia teoria stessa. Diana Iuele (University of Toronto), in "Insights on Italian Language Readers" alla luce delle teorie linguistiche sanzionate dal Council of Modern Languages Project, ha preso in esame alcuni dei testi piú rappresentativi di conversazione per corsi preparatori di italiano mettendone in evidenza i demeriti piú notevoli. Allo stesso tempo ha proposto un assetto metodologico atto a soddisfare efficientemente le esigenze funzionali e comunicative dell'insegnamento della lingua. Herbert J. Izzo (University of Calgary), in "Literary Standard, Regional Standards, and the Teaching of Italian Pronunciation in North America" ha sostenuto che la pronuncia dell'italiano standard (che differenzia le vocali "aperte" $\mathrm{e}$, o $[\epsilon$, J] di sfera, oro da quelle "chiuse" e, o [e, o] di pera, ora, e la s [z] sonora di rosa dalla $\mathrm{s}$ [s] sorda di cosa, e che raddoppia le consonanti dopo certe parole: la casa [lakása] vs a casa [akkása]) viene ora rimpiazzata da una pronuncia piú semplice (la nordica) piú vicina all'ortografia. Ha concluso che questa pronuncia piú semplice è quella che bisognerebbe insegnare agli studenti stranieri.

In conclusione le giornate di Guelph sono state utili e fruttuose sia per i risultati cui si è pervenuti sia per gli stimoli e le proposte di nuove ricerche che sono emerse, anche grazie ai numerosi interventi dei partecipanti. L'appuntamento per l'anno prossimo è a Montreal.

LEONARD G. SBROCCHI

University of Ottawa 